\title{
Forkhead box protein Al is a prognostic predictor and promotes tumor growth of gastric cancer
}

This article was published in the following Dove Press journal:

OncoTargets and Therapy

20 October 2015

Number of times this article has been viewed

\author{
Hongyu Ren ${ }^{1, *}$ \\ Pei Zhang 2 ,* \\ Yong Tang ${ }^{3}$ \\ Mengping $\mathrm{Wu}^{4}$ \\ Weikang Zhang ${ }^{2}$
}

'Department of Gastroenterology, Union Hospital, ${ }^{2}$ Department

of Gastrointestinal Surgery,

Union Hospital, ${ }^{3}$ Department of

Hepatobiliary Surgery, Union Hospital, ${ }^{4}$ Department of Internal Medicine,

Tongji Hospital, Tongji Medical

College, Huazhong University of

Science and Technology, Wuhan,

People's Republic of China

*These authors contributed equally to this work

Correspondence: Weikang Zhang

Department of Gastrointestinal

Surgery, Union Hospital, Tongji

Medical College, Huazhong University

of Science and Technology, No 1277

Jiefang Road, Wuhan 430022,

People's Republic of China

Tel +86278572 6560

Fax +862785726560

Email wkz_tongii@163.com
Abstract: Previous studies have demonstrated the cancer-type specific role of forkhead box protein A1 (FOXA1) in human malignancies. However, the clinical significance of FOXA1 and its biological function in gastric cancer remain unknown. In this study, the expression of FOXA1 in 80 pairs of gastric cancer tissues and corresponding non-tumor tissues was analyzed using immunohistochemistry and quantitative real-time polymerase chain reaction. We found that the levels of FOXA1 protein and mRNA in gastric cancer tissues were significantly higher than those in matched tumor-adjacent tissues. Furthermore, clinical association analysis indicated that the positive expression of FOXA1 was associated with adverse clinicopathological characteristics of gastric cancer patients including poor tumor differentiation, large tumor size, and advanced tumor-node-metastasis tumor stage. Notably, gastric cancer patients with positive expression of FOXA1 had a poorer 5-year overall survival and recurrence-free survival. In addition, FOXA1 knockdown remarkably inhibited cell proliferation and induced apoptosis in both SGC-7901 and MGC-803 cells. In vivo studies indicated that FOXA1 knockdown prominently suppressed tumor growth of gastric cancer in a nude mouse xenograft model. Mechanistically, we disclosed that the expression of Yes-associated protein was decreased accordingly after FOXA1 knockdown in both SGC-7901 and MGC-803 cells. Taken together, our data suggest that FOXA1 may serve as a promising prognostic indicator and an attractive therapeutic target of gastric cancer.

Keywords: FOXA1, gastric cancer, proliferation, apoptosis, YAP

\section{Introduction}

Gastric cancer is the fourth most common malignancy worldwide with a relatively higher incidence in eastern Asia region. ${ }^{1}$ And it is the third leading cause of cancer-related deaths, responsible for 723,000 deaths annually. ${ }^{2}$ The long-term prognosis of gastric cancer patients is still dismal with a less than $30 \%$ 5-year survival rate. ${ }^{3,4}$ The unsatisfactory prognosis of gastric cancer largely results from lack of effective biomarkers and targeted therapy. Therefore, it is important to elucidate the molecular mechanism involved in the development and progression of gastric cancer, and these will provide new avenues to identify novel biomarkers and therapeutic targets of gastric cancer, which may significantly improve the clinical outcomes of gastric cancer patients.

Forkhead box protein A1 (FOXA1), a member of forkhead box gene superfamily, is a pioneer transcription factor ${ }^{5}$ and plays pleiotropic roles in the development and differentiation. ${ }^{6-10}$ It induces the rearrangement of nucleosomal and alters the chromatin accessibility for other collaborating transcriptional regulators. ${ }^{5,11}$ In this way, FOXA1 regulates tissue-specific transcriptional programs and plays critical roles in cell growth, proliferation, apoptosis, and differentiation. ${ }^{11}$ Recently, emerging studies have focused on investigating the role of FOXA1 in human malignancies. ${ }^{5,7}$ Notably, FOXA1 was found to be overexpressed in anaplastic thyroid cancer, ${ }^{12}$ lung cancer, ${ }^{13,14}$ and esophageal 
cancer $^{13}$ suggesting the oncogenic roles of FOXA1 in human cancers. Nuclear staining of FOXA1 promoted cell proliferation of thyroid cancer ${ }^{12}$ and metastasis of prostate cancer ${ }^{15}$ and lung cancer. ${ }^{14}$ Otherwise, FOXA1 participated in the hepatocarcinogenesis of male mice and was responsible for the sexual dimorphism of hepatocellular carcinoma. ${ }^{16}$ However, in pancreatic cancer, the expression of FOXA1 in poorly differentiated tissues was significantly lower as compared with that in normal epithelium and precancerous lesions, ${ }^{17}$ suggesting a tumor suppressive role of FOXA1. Therefore, the role of FOXA1 in human malignancies seems to depend on the cancer type. However, the clinical significance of FOXA1 and its biological role in gastric cancer are still undefined.

In the present study, our results confirmed that the expression of FOXA1 was significantly upregulated in gastric cancer as compared with matched noncancerous tissues. The positive expression of FOXA1 was significantly correlated with adverse clinicopathological features and reduced survival of gastric cancer patients. Furthermore, we suggested that FOXA1 might promote gastric cancer cell proliferation and inhibit apoptosis partly by upregulating Yes-associated protein (YAP) expression.

\section{Materials and methods Patients and clinicopathological data}

A total of 80 pairs of clinical specimens including gastric cancer and matched tumor-adjacent tissues were obtained from patients who underwent curative gastrectomy in the Department of Gastrointestinal Surgery at Union Hospital during December 2007 to December 2009. All patients including 55 males and 25 females did not receive any radiotherapy or chemotherapy before surgical resection. All samples were collected and evaluated for FOXA1 expression after obtaining informed consent from patients. The clinicopathological data of these enrolled patients were collected from medical records and presented in Table 1. The protocols of this study were approved by the Huazhong University of Science and Technology Ethics Committee according to the Declaration of Helsinki (as revised in Tokyo 2004) (Permit Number: 2014-0065).

Table I Clinical association analysis of FOXAI expression in gastric cancer

\begin{tabular}{|c|c|c|c|c|}
\hline \multirow[t]{2}{*}{ Clinicopathological features } & \multirow[t]{2}{*}{ Total no of patients, $n=80$} & \multicolumn{2}{|l|}{ No of patients } & \multirow[t]{2}{*}{$P$-value } \\
\hline & & Positive FOXAI (\%) & Negative FOXAI (\%) & \\
\hline \multicolumn{5}{|l|}{ Age (years) } \\
\hline$<65$ & 41 & $29(70.7)$ & $12(29.3)$ & \multirow[t]{2}{*}{0.119} \\
\hline$\geq 65$ & 39 & $21(53.8)$ & I8 (46.2) & \\
\hline \multicolumn{5}{|l|}{ Sex } \\
\hline Male & 55 & $38(69.1)$ & $17(30.9)$ & \multirow[t]{2}{*}{0.071} \\
\hline Female & 25 & $12(48.0)$ & $13(52.0)$ & \\
\hline \multicolumn{5}{|l|}{ Histology } \\
\hline Well, moderate & 40 & $20(50.0)$ & $20(50.0)$ & \multirow[t]{2}{*}{$0.021 *$} \\
\hline Poor, signet & 40 & $30(75.0)$ & $10(25.0)$ & \\
\hline \multicolumn{5}{|l|}{ Size $(\mathrm{cm})$} \\
\hline$<5$ & 37 & $17(45.9)$ & $20(54.1)$ & \multirow[t]{2}{*}{$0.005^{*}$} \\
\hline$\geq 5$ & 43 & $33(76.7)$ & $10(23.3)$ & \\
\hline \multicolumn{5}{|l|}{ Depth } \\
\hline $\mathrm{T}_{1}$ & 19 & $10(52.6)$ & $9(47.4)$ & \multirow[t]{2}{*}{0.309} \\
\hline $\mathrm{T}_{2}-\mathrm{T}_{4}$ & 61 & $40(65.6)$ & $21(34.4)$ & \\
\hline \multicolumn{5}{|l|}{ Lymph node metastasis } \\
\hline Absent & 28 & $18(64.3)$ & $10(35.7)$ & \multirow[t]{2}{*}{0.809} \\
\hline Present & 52 & $32(61.5)$ & $20(38.5)$ & \\
\hline \multicolumn{5}{|l|}{ Lymphatic invasion } \\
\hline Absent & 21 & II (52.4) & $10(47.6)$ & \multirow[t]{2}{*}{0.265} \\
\hline Present & 59 & $39(66.1)$ & $20(33.9)$ & \\
\hline \multicolumn{5}{|l|}{ Venous infiltration } \\
\hline Absent & 57 & $36(63.2)$ & $21(36.8)$ & \multirow[t]{2}{*}{0.848} \\
\hline Present & 23 & $14(60.9)$ & $9(39.1)$ & \\
\hline \multicolumn{5}{|l|}{ TNM stage } \\
\hline I, II & 50 & $27(54.0)$ & $23(46.0)$ & \multirow[t]{2}{*}{$0.043^{*}$} \\
\hline III, IV & 30 & $23(62.5)$ & 7 (37.5) & \\
\hline
\end{tabular}

Note: *Statistically significant.

Abbreviations: FOXAI, forkhead box protein AI; TNM, tumor-node-metastasis; no, number. 


\section{Immunohistochemical staining}

Formalin-fixed samples were embedded in paraffin and cut into $4 \mu \mathrm{m}$ thick sections. The sections were deparaffinized using xylene and rehydrated through graded ethanol. Antigen retrieval was conducted and heated at boiling point for 2 minutes. Endogenous peroxidase activity of these slides was quenched by incubation with $3 \%$ hydrogen peroxide for 10 minutes. After incubating with $5 \%$ of bovine serum albumin for 10 minutes, these sections were incubated overnight at $4^{\circ} \mathrm{C}$ with primary antibody against FOXA1 (1:100, \#5089, Abcam, Cambridge, MA, USA) or Ki-67 (1:100, \#9027, Cell Signaling, Danvers, MA, USA). The biotinylated secondary antibody (ZSGB-Bio, Beijing, People's Republic of China) was used to detect the primary antibody. Then sections were incubated with diaminobenzidine and counterstained with hematoxylin. Finally, they were dehydrated in graded ethanol and transparentized in xylene. The percentage of positive tumor cells was graded as per the following criteria: 0 , less than $10 \%$; $1,10 \%-30 \%$; $2,31 \%-50 \%$; and 3 , more than $50 \%$.

\section{Cell culture and transfection}

Human gastric cancer cell lines, SGC-7901 and MGC-803, were purchased from the Shanghai Institute of Biochemistry and Cell Biology, Chinese Academy of Sciences (Shanghai, People's Republic of China) for in vitro experiments. Cells were cultured in Dulbecco's modified Eagle's medium (DMEM, Gibco, Grand Island, NY, USA) containing 10\% fetal bovine serum (Gibco) with 100 units/mL penicillin and $100 \mu \mathrm{g} / \mathrm{mL}$ streptomycin. All cells were maintained in a $5 \%$ $\mathrm{CO}_{2}$ atmosphere at $37^{\circ} \mathrm{C}$.

The targeted sequences for FOXA1 small interfering RNA (sense 5'-GCACUGCAAUACUCGCCUU-3') or a nonspecific duplex oligonucleotide as a negative control were synthesized by Sangon Biotech (Shanghai) Co., Ltd. (Shanghai, People's Republic of China). The non-targeting vector (TR30012, OriGene, Beijing, People's Republic of China) or the FOXA1-specific short hairpin RNA (shRNA) (TR312942, OriGene) was transfected into gastric cancer cells using Lipofectamine 2000 following the manufacturer's instructions (catalog number: 11668-027, Thermo Fisher Scientific, Waltham, MA, USA). The cells were collected for further experiments 48 hours after transfection.

\section{Immunoblotting}

Cells were lysed in RIPA buffer (50 mM Tris $\mathrm{pH} 7.5$, $150 \mathrm{mM} \mathrm{NaCl}, 1 \%$ TritonX-100, $5 \mathrm{mM}$ ethylenediaminetetraacetic acid) supplemented with inhibitors of proteases.
Protein concentration was measured by the BCA Kit (Pierce, Rockford, IL, USA). Protein samples $(20 \mu \mathrm{g})$ were separated by sodium dodecyl sulfate polyacrylamide gel electrophoresis and transferred onto nitrocellulose membranes. The polyvinylidene fluoride membranes were then incubated with antibodies against the following primary antibodies: FOXA1 (1:1000), YAP (1:1000,\#12395, Cell Signaling), Caspase-3 (1:1000, \#9662, Cell Signaling), and glyceraldehyde 3-phosphate dehydrogenase (1:1000, \#2118, Cell Signaling). After washing the membranes three times with Tris-Buffered Saline Tween-20, blots were probed with horseradish peroxidase-conjugated secondary antibodies (1:10000, Bio-Rad Laboratories Inc., Hercules, CA, USA) and detected using the HyGLO Horse reddish peroxidase detection kit.

\section{Real-time quantitative reverse transcription polymerase chain reaction}

Total RNA was extracted from clinical specimens using TRIzol based on the manufacture's protocol (Invitrogen). Reverse transcription was performed using a Thermo Scientific RevertAid Premium First Strand cDNA Synthesis Kit (Thermo Scientific, Rockford, IL, USA). Power SYBR ${ }^{\circledR}$ Green PCR Master Mix (Thermo Scientific) was employed to perform cDNA amplification. Specific primers to detect the expression levels of FOXA1 and YAP included: FOXA1 sense primer $5^{\prime}$-AGGGCTGGATGGTATTG-3' and antisense primer $5^{\prime}$-ACCGGGACGGAGGAGTAG-3'; YAP sense primer $5^{\prime}$-CCTGCGTAGCCAGTTACCAA- $3^{\prime}$ and antisense primer $5^{\prime}$-CCATCTCATCCACACTGTTC-3'. GAPDH gene was used as an internal control. The primers of GAPDH were $5^{\prime}$-CGGATTTGGTCGTATTGG-3' and 5'-TCCTGGAAGATGGTGATG-3'. The relative expression of FOXA1 or YAP was normalized to internal control. Three separate experiments were conducted for each clone.

\section{Proliferation and apoptosis assay}

An amount of $5 \times 10^{3}$ gastric cancer cells per well were seeded into 96-well plates. The proliferation assay was assessed based on the instruction of the BrdU ELISA kit (Roche, Indianapolis, IN, USA). The percentage of apoptotic cells were investigated based on the instruction of Annexin-VFLUOS Staining Kit (Roche). Briefly, $1 \times 10^{5}$ cells were seeded in six-well plates and cultured for 24 hours. The cells were collected and resuspended in $100 \mu \mathrm{L}$ binding buffer. Then, the cells were incubated with $5 \mu \mathrm{L}$ fluorescein isothiocyanate-Annexin- $\mathrm{V}$ in the dark for 15 minutes at room temperature. Subsequently, $5 \mu \mathrm{L}$ PI was added and incubated 
with the cells for 20 minutes at room temperature in the dark. Finally, the cell samples were examined in the flow cytometer. Each assessment of proliferation and apoptosis was repeated three times.

\section{In vivo experiments}

An amount of $3 \times 10^{6}$ SGC-7901 cells transfected with nontargeting shRNA or FOXA1 shRNA were resuspended in $100 \mu \mathrm{L}$ of phosphate buffer saline and consequently injected subcutaneously into the right dorsal flank of 4- to 6-week-old male nude mice. Tumor volume was measured with calipers every 3 days, and then calculated as tumor volume $=$ length $\times$ width $\times$ width $/ 2$. All mice were sacrificed at 3 weeks after the injection of SGC-7901 cells. The xenograft tumor tissues were isolated for pathological examination. Apoptosis cells in the isolated tumor tissues was detected using a TUNEL assay kit (4810-30-K, R\&D Systems, Inc., Minneapolis, MN, USA) based on the manufacturer's guidelines. All in vivo experiments protocols were approved by the Institutional Animal Care and Use Committee of Huazhong University of Science and Technology.

\section{Statistical analysis}

The quantitative data were compared between groups using the Student's $t$-test or analysis of variance (ANOVA). Categorical data were analyzed using the Pearson's $\chi^{2}$ test. The cumulative recurrence and survival rates were determined using the Kaplan-Meier method and log-rank test. A value of $P<0.05$ was considered to be significant. All the analyses were performed using the SPSS statistical package for Windows Version 13 (SPSS Inc., Chicago, IL, USA) or GraphPad Prism 5 software (GraphPad Software, Inc., La Jolla, CA, USA).

\section{Results}

\section{FOXAI expression is elevated in gastric cancer tissues}

Immunohistochemical staining was performed to investigate the expression of FOXA1 between gastric cancer tissues and matched tumor-adjacent tissues. As shown in Figure 1, negative staining of FOXA1 was observed in adjacent noncancerous tissue (Figure 1A), while positive staining of FOXA1 with nuclear location was presented in gastric cancer tissues (Figure 1B-D). The comparison of immunohistochemistry scores indicated that the level of FOXA1 protein in gastric cancer tissues was significantly upregulated as compared with adjacent noncancerous tissues $(P<0.05$, Figure 2A). Furthermore, 20 randomly selected cases were subjected to quantitative reverse transcription polymerase chain reaction for FOXA1 mRNA. We found the expression of FOXA1 mRNA was significantly higher in gastric cancer tissues than that in corresponding tumor-adjacent tissues $(P<0.05$, Figure $2 \mathrm{~B})$. There results indicate an oncogenic role of FOXA1 in gastric cancer.

\section{Positive expression of FOXAI was associated with poor clinicopathological features}

To elucidate the clinical significance of FOXA1 expression in gastric cancer, we investigated the relationship between FOXA1 expression and clinicopathological features of the gastric cancer patients. The immunoreactivity of FOXA1 was considered as either negative (score 0 ) or positive (scores 1-3). As shown in Table 1, positive expression of FOXA1 in gastric cancer tissues was associated with poor tumor differentiation $(P=0.021)$, large tumor size $(P=0.005)$, and advanced tumor-node-metastasis stage $(P=0.043)$. These results indicate that FOXA1 may promote the development and progression of gastric cancer.

\section{FOXAI is a prognostic predictor for gastric cancer patients}

To further investigate the prognostic value of FOXA1 expression, the overall survival and the recurrence-free survival rates were compared between the FOXA1 positive $(n=50)$ and FOXA1 negative groups $(n=30)$. Kaplan-Meier survival curves showed that positive expression of FOXA1 in gastric cancer was significantly correlated with poorer overall survival $(P=0.002$, Figure 3$)$ and recurrence-free survival rates ( $P=0.007$, Figure 3$)$. These data indicate that FOXA1 expression in gastric cancer is a potent predictor of patients' prognosis.

\section{FOXAI knockdown inhibits gastric cancer cell proliferation and promotes apoptosis in vitro and in vivo}

To determine the underlying role of FOXA1 in gastric cancer, a specific FOXA1 shRNA was used to inhibit the expression of FOXA1 in SGC-7901 cells, which showed a relative higher basal expression of FOXA1. FOXA1 knockdown was confirmed by quantitative reverse transcription polymerase chain reaction and immunoblotting $(P<0.05$, respectively, Figure 4A and B). Subsequently, BrdU incorporation assays showed that the proliferation of SGC-7901 cells was significantly decreased after FOXA1 knockdown $(P<0.05$, Figure $4 \mathrm{C})$. Otherwise, the percentage of apoptotic SGC-7901 cells was significantly increased after downregulation of FOXA1 $(P<0.05$, Figure 4D). Western blot analyses found that FOXA1 knockdown evidently 

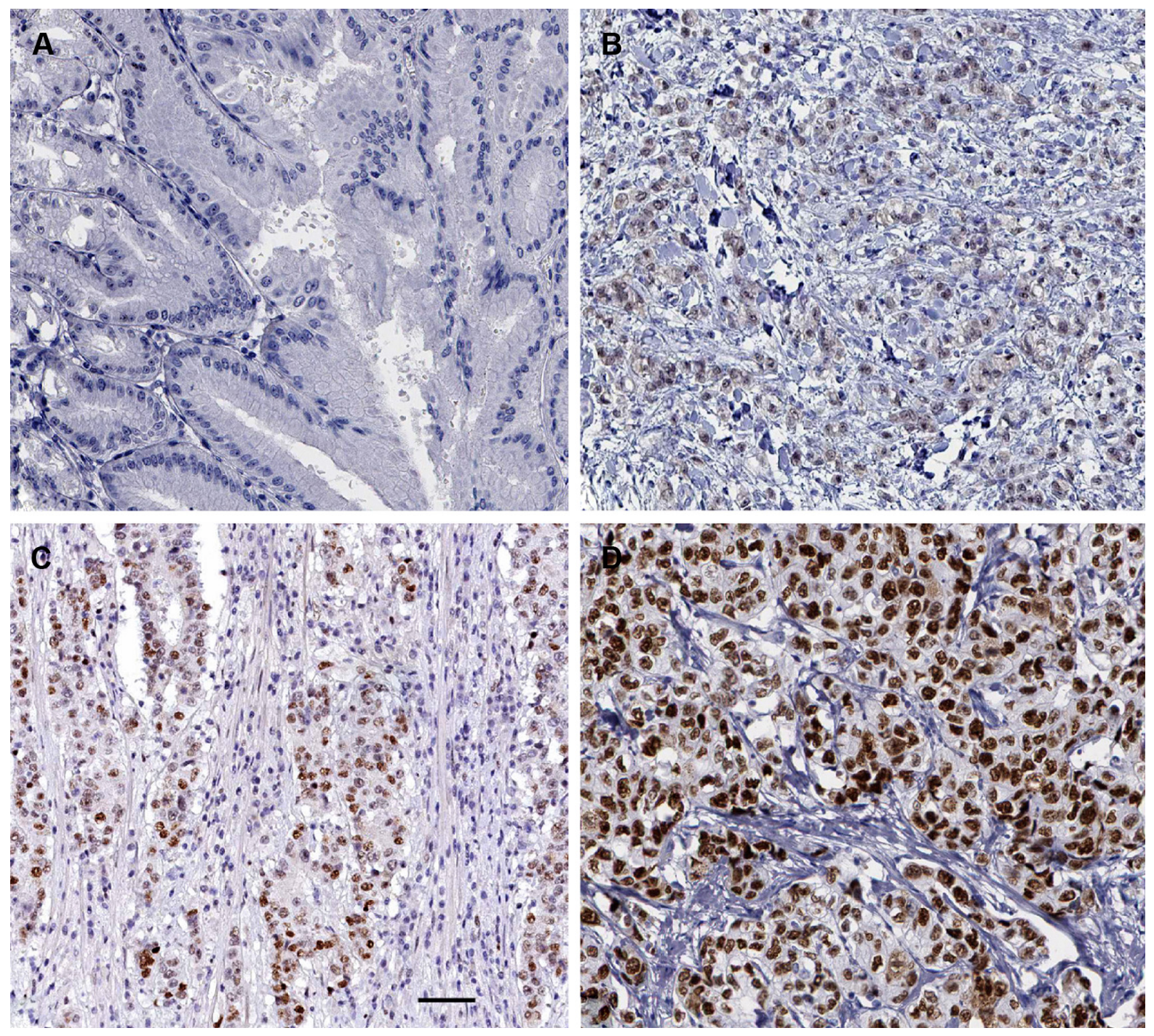

Figure I Immunohistochemical staining of FOXAI in tumor-adjacent tissues and gastric cancer tissues.

Notes: (A) Negative staining of FOXAI in the tumor-adjacent tissues; (B) Low, (C) medium, and (D) high expression of FOXAI in gastric cancer tissues. Scale bar: $50 \mu \mathrm{m}$. Abbreviation: FOXAI, forkhead box protein AI.

A

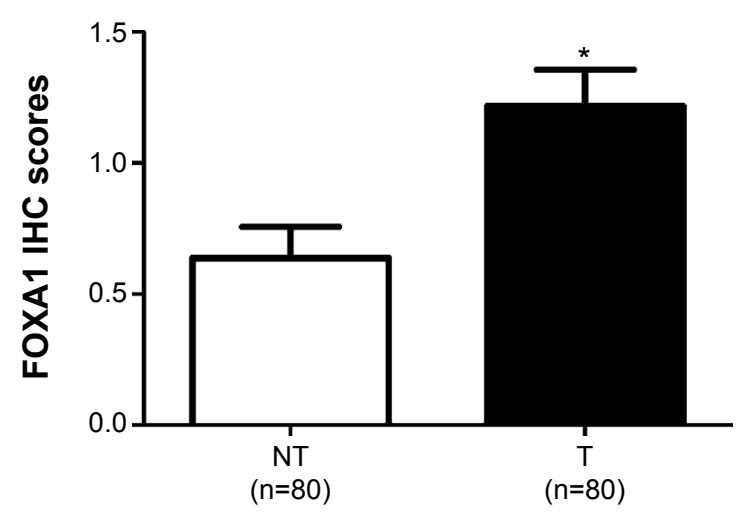

B

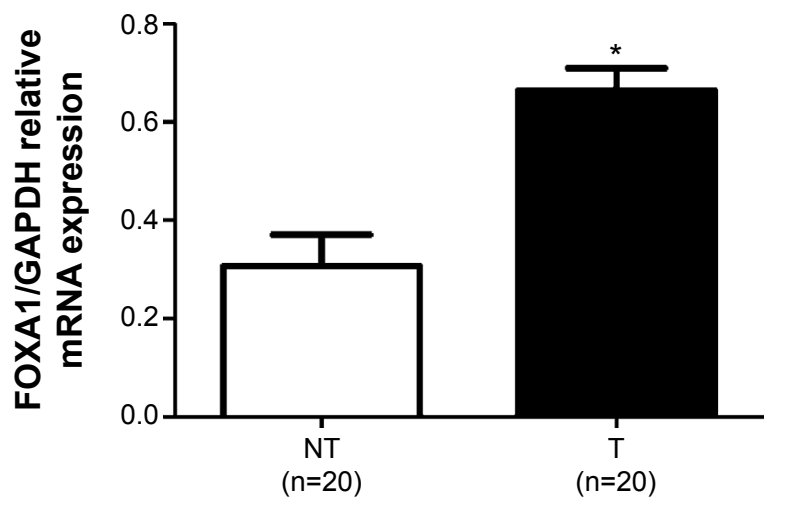

Figure 2 Expression levels of FOXAI in gastric cancer tissues (T) and matched non-tumor tissues (NT).

Notes: (A) Comparing differences in the expression levels of FOXAI protein between gastric cancer tissues (T) and matched non-tumor tissues (NT). (B) qRT-PCR demonstrated that the mRNA level of FOXAI in gastric cancer tissues was significantly increased as compared with that in matched non-tumor tissues. $* P<0.05$ by $t$-test. Abbreviations: FOXAI, forkhead box protein AI; NT, non-tumor tissues; qRT-PCR, quantitative reverse transcription polymerase chain reaction. 

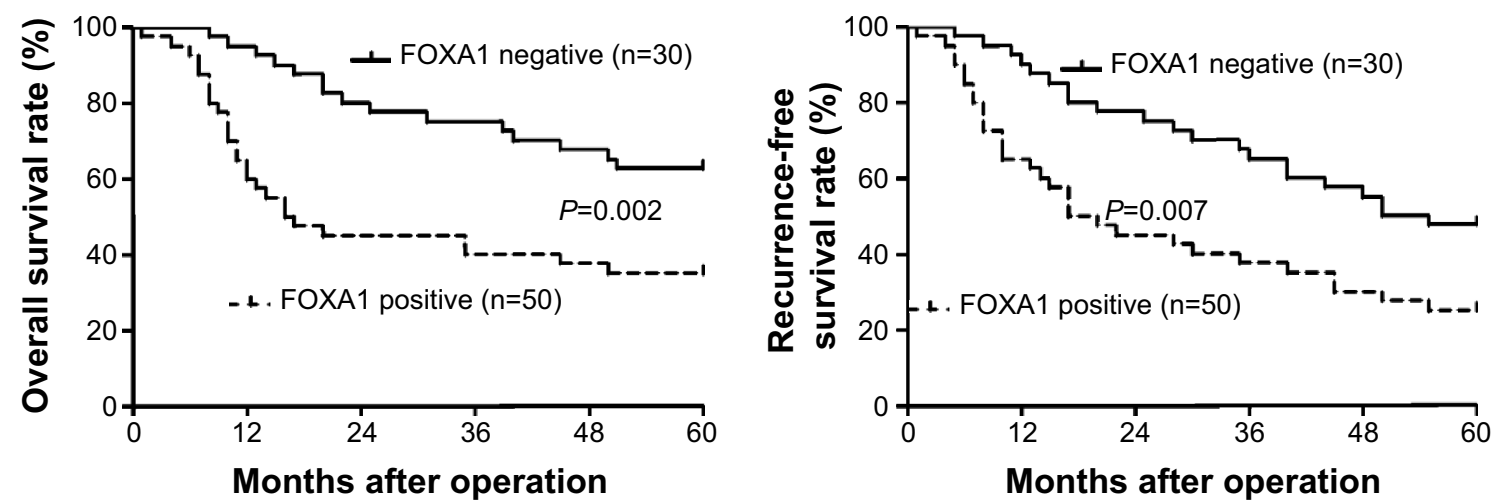

Figure 3 Prognostic value of FOXAI for gastric cancer patients.

Notes: Gastric cancer patients were divided into FOXAI negative $(n=30)$ and positive groups $(n=50)$ according to the immunostaining scores. Both the overall survival (left panel) and recurrence-free survival rates (right panel) in the FOXAI positive group were significantly reduced as compared with those in FOXAI negative group. Abbreviation: FOXAI, forkhead box protein AI.

increased the expression of cleaved Caspase-3 protein in SGC-7901 cells $(P<0.05$, Figure 4E). Notably, the effects of FOXA1 shRNA on gastric cancer cell proliferation and apoptosis were confirmed by a specific small interfering RNA targeting FOXA1 (data not shown). Furthermore, MGC-803 cells with FOXA1 knockdown were established $(P<0.05$, respectively, Figure 5A and B). Similarly, BrdU incorporation and flow cytometry assays indicated that FOXA1 knockdown inhibited cell proliferation and induced apoptosis in MGC-803 cells $(P<0.05$, Figure 5C-E).

Next, SGC-7901 cells that were transfected with nontargeting shRNA or FOXA1 shRNA were subcutaneously implanted into nude mice. As shown in Figure 6A, FOXA1 knockdown significantly inhibited the growth of SGC-7901 cells in nude mice $(P<0.05)$. Moreover, Ki-67 staining and TUNEL assays showed that FOXA1 knockdown significantly inhibited SGC-7901 cell proliferation and promoted apoptosis in vivo $(P<0.05$, respectively, Figure $6 \mathrm{~B}$ and $\mathrm{C})$.

\section{FOXAI repression inhibits the expression of YAP in gastric cancer cells}

Hippo-YAP signaling has been confirmed to play a fundamental role in the pathogenesis of gastric cancer. ${ }^{18,19}$ And inhibition of YAP expression led to a remarkable decrease of cell proliferation and increase of apoptosis in gastric cancer cells. ${ }^{20} \mathrm{~A}$ recent study demonstrated that opening the compacted chromatin by FOXA1 around cAMP response element binding protein (CREB) binding site within the YAP promoter facilitates CREB-mediated YAP transcription in hepatocellular carcinoma. ${ }^{21}$ Therefore, we investigated whether the effects of FOXA1 on gastric cancer cells were mediated via modulating YAP expression. Gastric cancer cell lines, SGC-7901 and MGC-803, were subjected to immunoblotting after FOXA1 knockdown. As expected, FOXA1 knockdown resulted in a significant decrease of YAP expression in both mRNA and protein levels in both SGC-7901 and MGC-803 cells $(P<0.05$, Figure 7$)$. These data indicate FOXA1 may regulate the expression of YAP in gastric cancer cells.

\section{Discussion}

Treatment of advanced gastric cancer is a challenge for the physician. Currently, molecular-targeted drugs such as Volitinib and Apatinib were applied to treat advanced gastric cancer and achieved a better clinical outcome for patients. ${ }^{22,23}$ Thus, it is critical to identify novel biomarkers and therapeutic targets for the diagnosis and treatment of gastric cancer. In this study, we investigated the expression status of FOXA1 in gastric cancer for the first time. Significant elevated expression of FOXA1 in both mRNA and protein levels were observed in the gastric cancer tissues as compared with those in matched tumor-adjacent tissues. And it was more important to disclose that positive expression of FOXA1 was correlated with adverse clinicopathological features and poor prognosis of gastric cancer patients. Therefore, FOXA1 can potentially serve as a novel biomarker with a remarkable value in predicting the clinical outcome of gastric cancer patients.

The potential oncogenic role of FOXA1 in gastric cancer promoted us to investigate its biological role. Previous studies $^{24,25}$ have confirmed that FOXA1 was a forkhead transcription factor that regulated the chromatin structure and recruited other transcription factors to promote transcription of downstream targets. Functionally, FOXA1 was reported to be an important regulator of cell proliferation, cell cycle, and apoptosis. ${ }^{26-28}$ In our study, both in vitro and in vivo experiments demonstrated that FOXA1 knockdown inhibited cell 

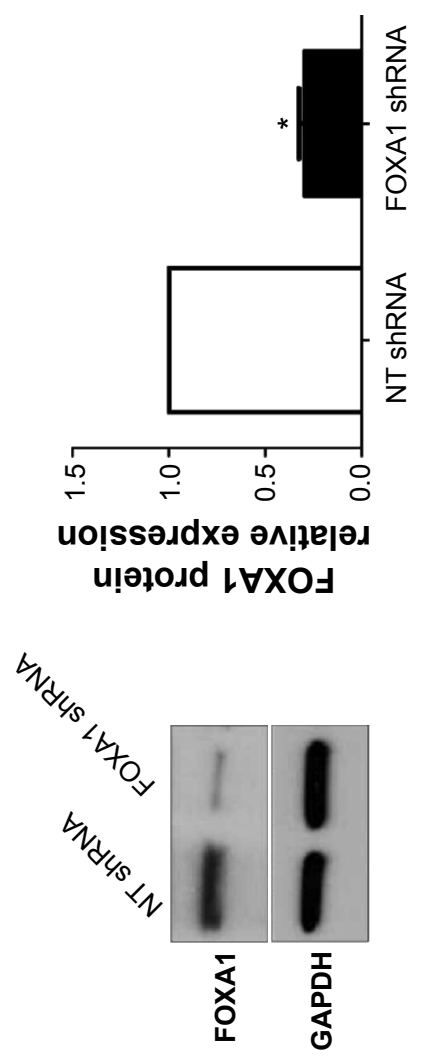

$\mathbf{m}$

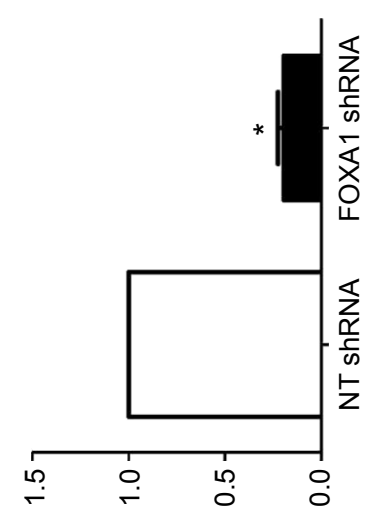

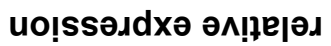

$\varangle \quad \forall N Y$ I $\forall$ XOJ
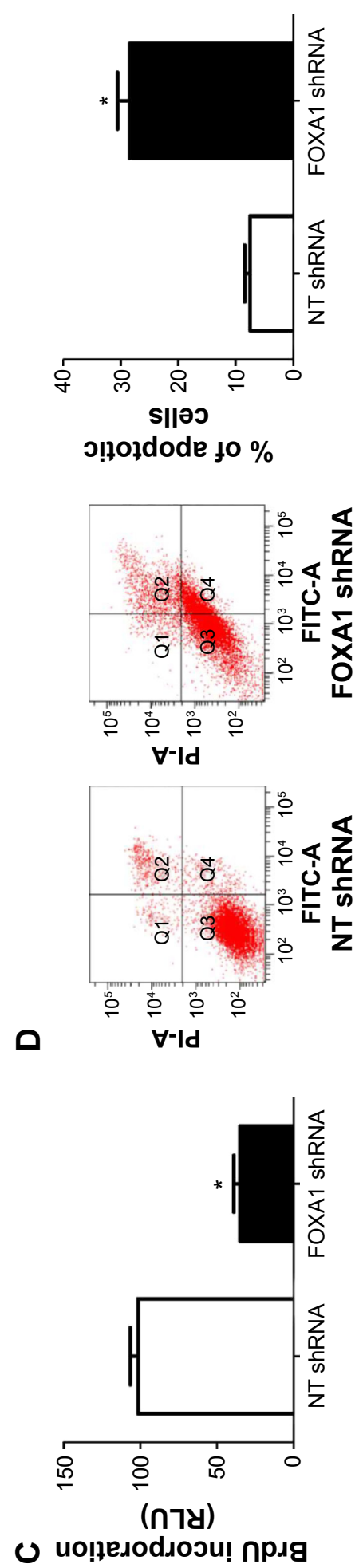

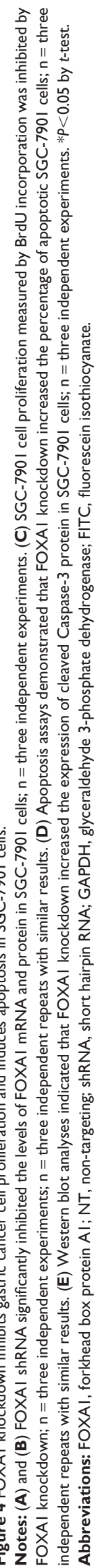




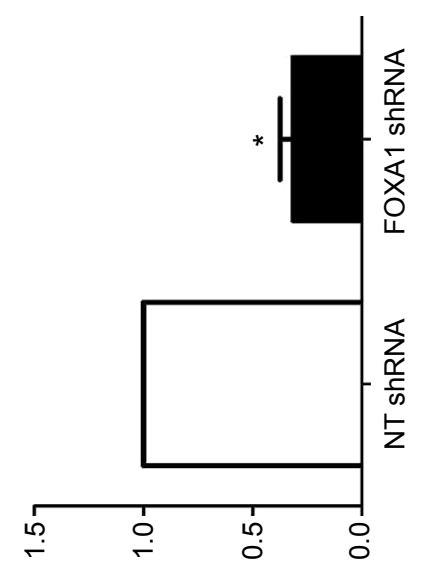

uo!ssəлdxә әм!ฺ리 u!ofold LXXO」
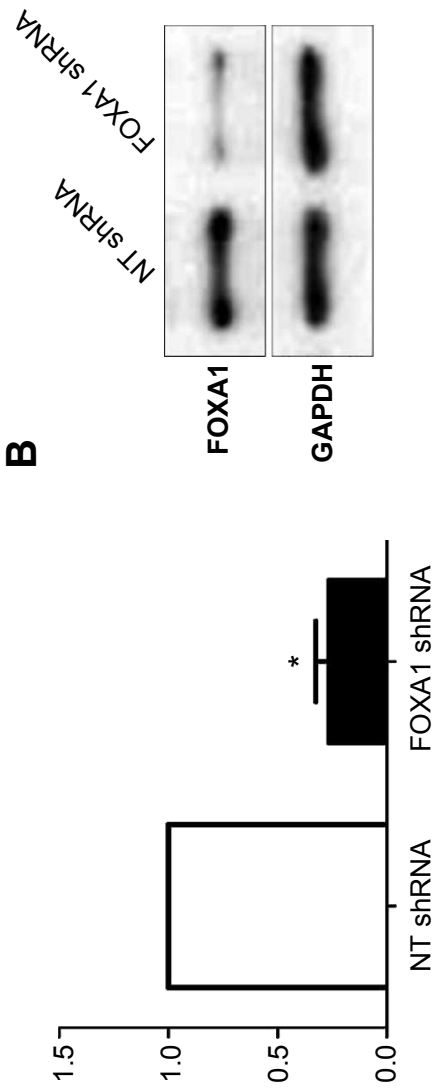

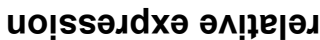

$\forall N Y W$ เ $\forall X O$
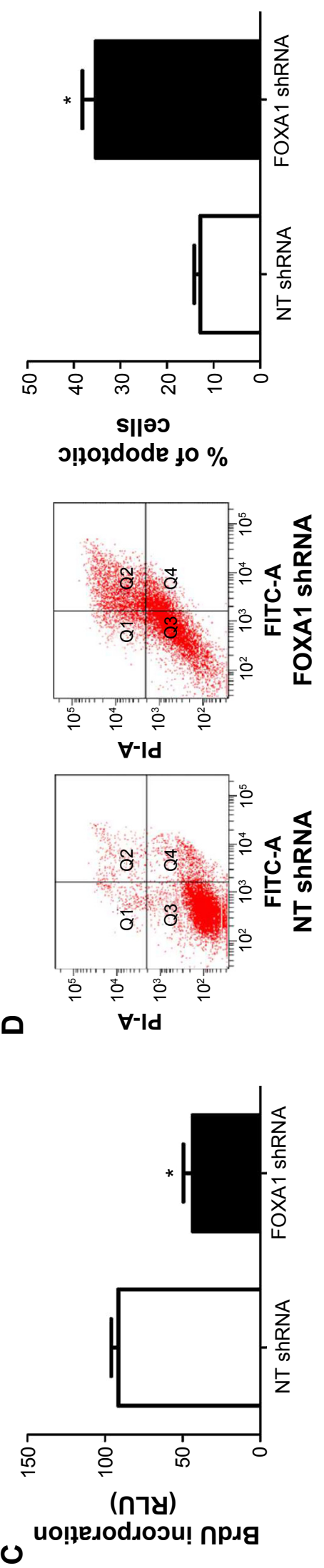

总

赔

을

문

气.

ํํำ

วิ

勇

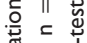

要

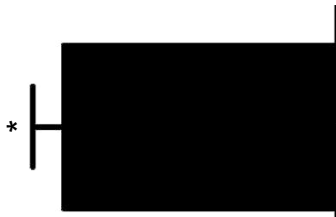

.

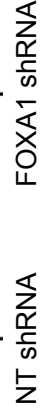

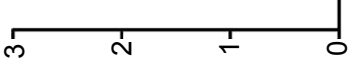

uolssə.ıdxə

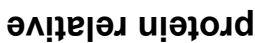
ع-әsedseว рәлеәэ

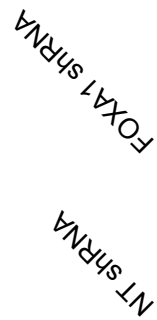

U

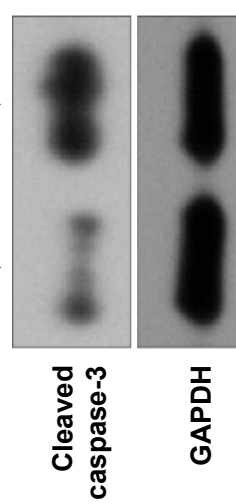
言囟

ब्ष

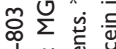

Uू

Uิ

的范泀

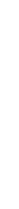

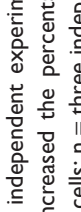

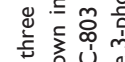

II
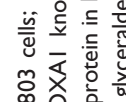

ư

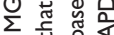

$\subseteq$ 卷

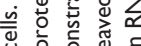

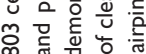

نㄴำ

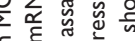

品文高

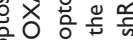

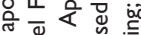

巡

홓

琙

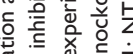

罂

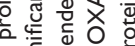

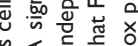

는 $\varangle$

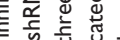

乡ँ $\mid 1$.

论芒㟧

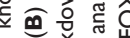

范哭 능

许这

中 $\ddot{0}$ Oิ

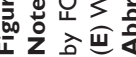


A
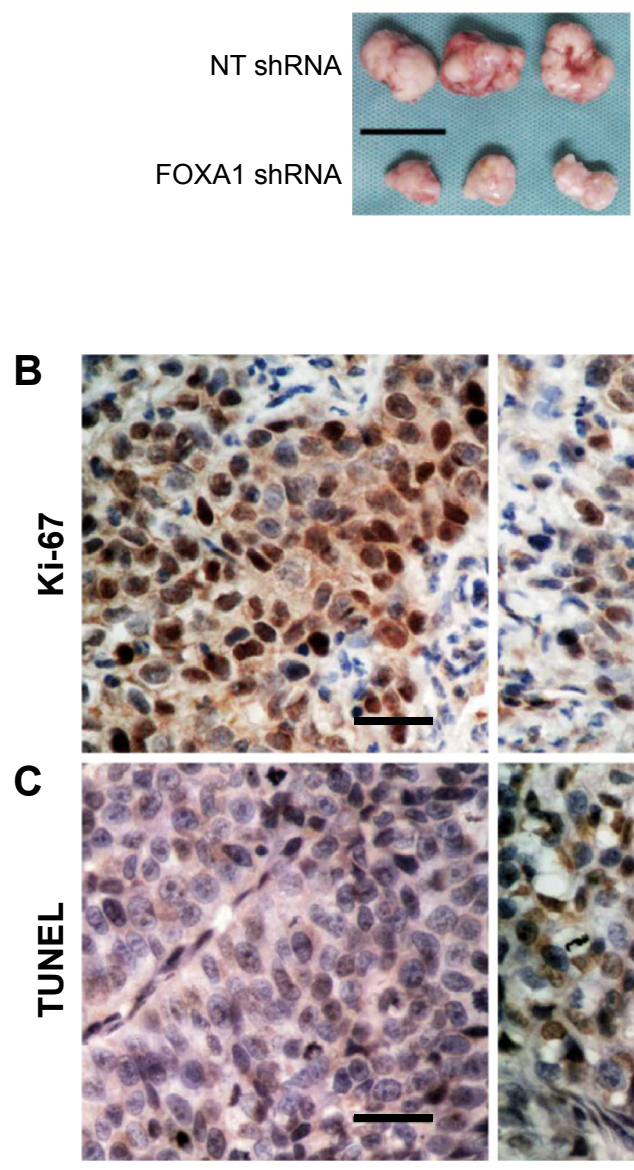

NT ShRNA
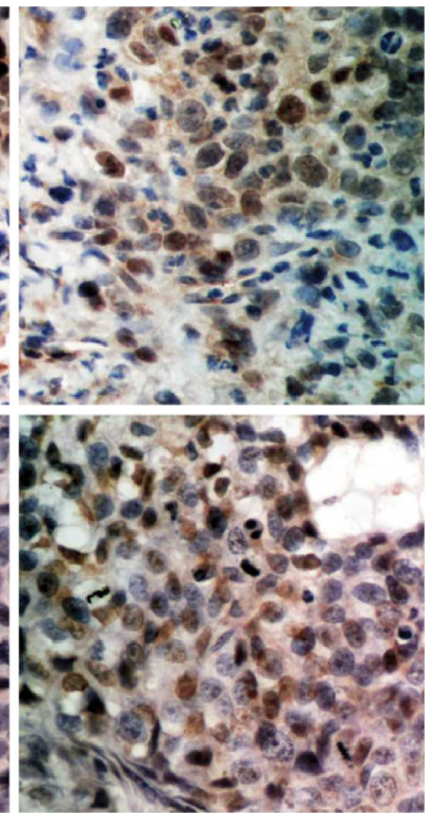

FOXA1 shRNA
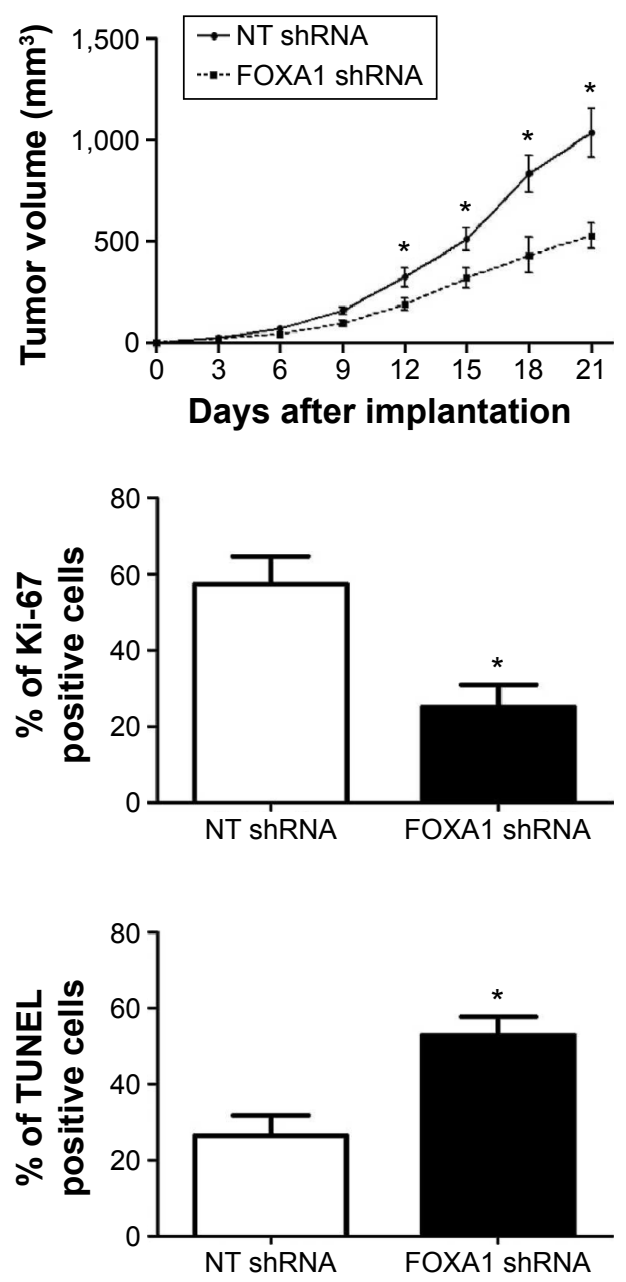

Figure 6 FOXAI knockdown suppresses tumor growth in nude mice.

Notes: (A) SGC-790I cells that were transfected with FOXAI shRNA or NT shRNA were injected subcutaneously into nude mice. Tumor growth curves showed that SGC-790I cells with FOXAI knockdown $(n=6)$ exhibited a significant slower growth as compared with control cells $(n=6)$. $* P<0.05$ by $A N O V A$. Scale bar: I cm. (B) Representative immunostaining of $\mathrm{Ki}-67$ and quantitative analysis of Ki-67 positive cells showed that FOXAI knockdown significantly reduced cell proliferation; $\mathrm{n}=6$, $* P<0.05$ by $t$-test. Scale bar: $20 \mu \mathrm{m}$. (C) Representative staining of TUNEL and quantitative analysis of TUNEL positive cells revealed that FOXAI knockdown significantly increased the number of apoptotic cells; $n=6, * P<0.05$ by $t$-test. Scale bar: $20 \mu \mathrm{m}$.

Abbreviations: FOXAI, forkhead box protein AI; NT, non-targeting; shRNA, short hairpin RNA; ANOVA, analysis of variance; TUNEL, terminal deoxynucleotidyl transferase dUTP nick end labeling.

proliferation and induced apoptosis in gastric cancer cells. Therefore, our data reveal that FOXA1 plays an oncogenic role in gastric cancer by promoting cell proliferation and preventing apoptosis.

Hippo-YAP signaling pathway has been found to play a critical role in gastric cancer. ${ }^{18-20,29}$ The expression of YAP has been confirmed to be significantly higher as compared with matched normal gastric mucosa in prior studies. ${ }^{30-32}$ And YAP regulates proliferation and apoptosis of gastric cancer cells. ${ }^{20}$ Thus, YAP has been regarded as a therapeutic target of gastric cancer. ${ }^{33}$ Interestingly, a recent study of hepatocellular carcinoma found that FOXA1 could open the compacted chromatin around CREB binding site within the YAP promoter, facilitated CREB-mediated YAP transcription, and thus resulted in increased expression of YAP in hepatocellular carcinoma cells. ${ }^{21}$ Therefore, we speculated that FOXA1 might exert its regulating effects on the proliferation and apoptosis of gastric cancer cells by modulating the expression of YAP. After repression of FOXA1 expression in gastric cancer cells with FOXA1-specific shRNA, the level of FOXA1 mRNA and protein was significantly decreased. These results suggest that FOXA1 may regulate cell proliferation and apoptosis at least in part through modulating YAP expression in gastric cancer cells.

\section{Conclusion}

The present study demonstrates for the first time that FOXA1 is overexpressed in gastric cancer. The positive expression of FOXA1 is associated with poor prognostic features and reduced survival of gastric cancer patients. Furthermore, FOXA1 plays an oncogenic role in gastric cancer by promoting cell proliferation and inhibiting apoptosis. FOXA1 
A

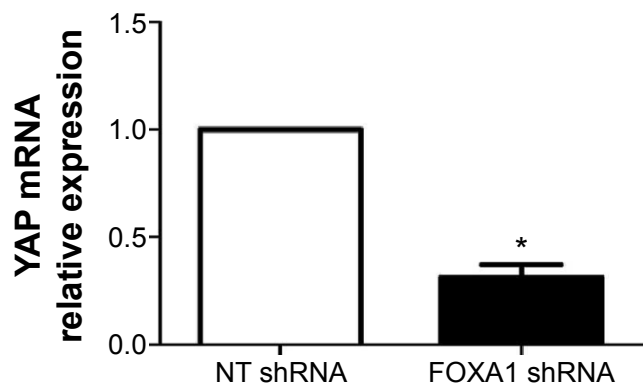

C

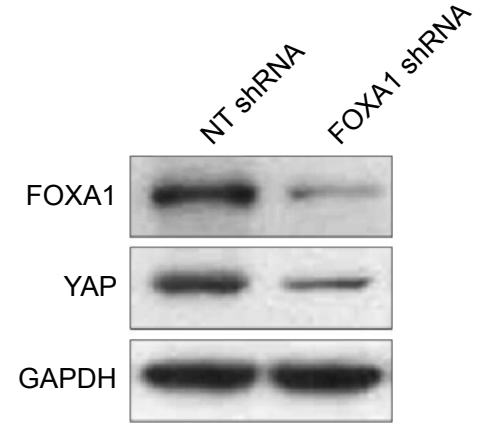

D

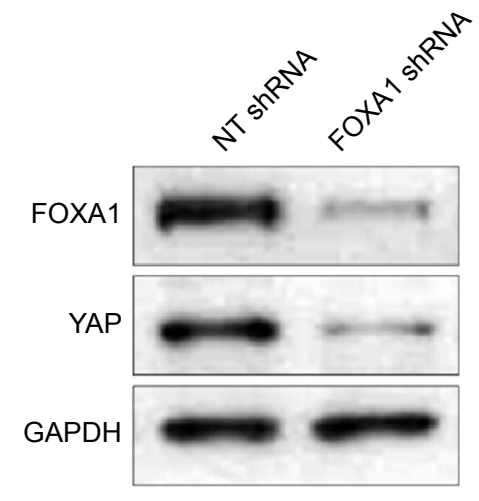

B MGC-803

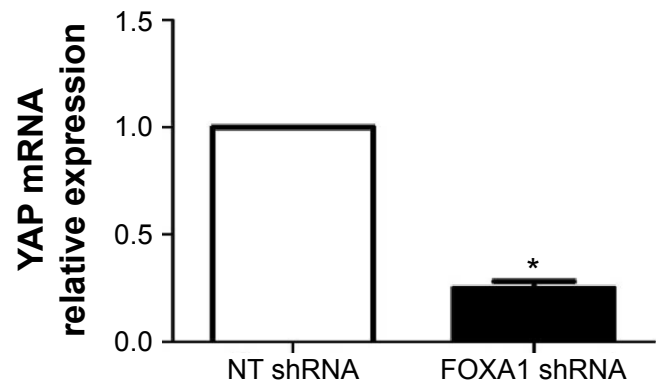

SGC-7901

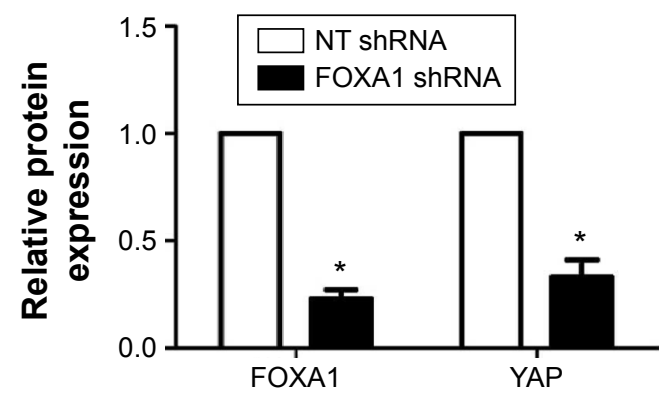

MGC-803

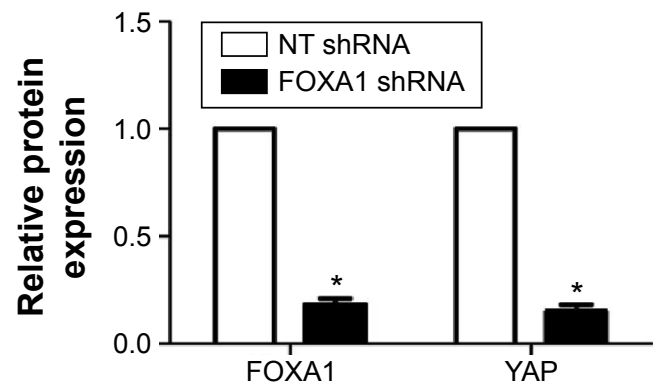

Figure 7 Downregulation of FOXAI reduces the expression of YAP in gastric cancer cells.

Notes: SGC-790I and MGC-803 cells that were transfected with FOXAI shRNA or NT shRNA were subjected to qRT-PCR and Western blot for YAP expression. (A) and (B) FOXAI knockdown significantly reduced the level of YAP mRNA in both SGC-790I and MGC-803 cells. (C) and (D) Inhibition of FOXAI clearly decreased the expression of YAP protein in both SGC-790I and MGC-803 cells; $n=$ three independent repeats with similar results. $* P<0.05$ by $t$-test.

Abbreviations: FOXAI, forkhead box protein AI; YAP, Yes-associated protein; qRT-PCR, quantitative reverse transcription polymerase chain reaction; NT, Non-targeting; shRNA, short hairpin RNA; GAPDH, glyceraldehyde 3-phosphate dehydrogenase.

may facilitate tumor growth of gastric cancer by modulating YAP. Taken together, this study indicates that FOXA1 may be a potent prognostic biomarker and can potentially serve as a therapeutic target of gastric cancer.

\section{Disclosure}

The authors report no conflicts of interest in this work.

\section{References}

1. Ferro A, Peleteiro B, Malvezzi M, et al. Worldwide trends in gastric cancer mortality (1980-2011), with predictions to 2015, and incidence by subtype. Eur J Cancer. 2014;50(7):1330-1344.
2. Ferlay J, Soerjomataram I, Dikshit R, et al. Cancer incidence and mortality worldwide: sources, methods and major patterns in GLOBOCAN 2012. Int J Cancer. 2015;136(5):E359-E386.

3. Herszenyi L, Tulassay Z. Epidemiology of gastrointestinal and liver tumors. Eur Rev Med Pharmacol Sci. 2010;14(4):249-258.

4. Brenner H, Rothenbacher D, Arndt V. Epidemiology of stomach cancer. Methods Mol Biol. 2009;472:467-477.

5. Bernardo GM, Keri RA. FOXA1: a transcription factor with parallel functions in development and cancer. Biosci Rep. 2012;32(2):113-130.

6. Augello MA, Hickey TE, Knudsen KE. FOXA1: master of steroid receptor function in cancer. EMBO J. 2011;30(19):3885-3894.

7. Katoh M, Igarashi M, Fukuda H, Nakagama H, Katoh M. Cancer genetics and genomics of human FOX family genes. Cancer Lett. 2013;328(2): 198-206.

8. Strazzabosco M. Foxa1 and Foxa2 regulate bile duct development in mice. J Hepatol. 2010;52(5):765-767. 
9. Liu Z, Merkurjev D, Yang F, et al. Enhancer activation requires transrecruitment of a mega transcription factor complex. Cell. 2014;159(2): 358-373.

10. Hurtado A, Holmes KA, Ross-Innes CS, Schmidt D, Carroll JS. FOXA1 is a key determinant of estrogen receptor function and endocrine response. Nat Genet. 2011;43(1):27-33.

11. Zaret KS, Carroll JS. Pioneer transcription factors: establishing competence for gene expression. Genes Dev. 2011;25(21):2227-2241.

12. Nucera C, Eeckhoute J, Finn S, et al. FOXA1 is a potential oncogene in anaplastic thyroid carcinoma. Clin Cancer Res. 2009;15(11): 3680-3689.

13. Lin L, Miller CT, Contreras JI, et al. The hepatocyte nuclear factor 3 alpha gene, HNF3alpha (FOXA1), on chromosome band 14q13 is amplified and overexpressed in esophageal and lung adenocarcinomas. Cancer Res. 2002;62(18):5273-5279.

14. Deutsch L, Wrage M, Koops S, et al. Opposite roles of FOXA1 and NKX2-1 in lung cancer progression. Genes Chromosomes Cancer. 2012; 51(6):618-629.

15. Gerhardt J, Montani M, Wild P, et al. FOXA1 promotes tumor progression in prostate cancer and represents a novel hallmark of castrationresistant prostate cancer. Am J Pathol. 2012;180(2):848-861.

16. Anson M, Crain-Denoyelle A-M, Baud V, et al. Oncogenic $\beta$-catenin triggers an inflammatory response that determines the aggressiveness of hepatocellular carcinoma in mice. J Clin Invest. 2012;122(2): 586-599.

17. Song Y, Washington MK, Crawford HC. Loss of FOXA1/2 is essential for the epithelial-to-mesenchymal transition in pancreatic cancer. Cancer Res. 2010;70(5):2115-2125.

18. Zhou GX, Li XY, Zhang Q, et al. Effects of the hippo signaling pathway in human gastric cancer. Asian Pac J Cancer Prev. 2013;14(9): 5199-5205.

19. Cui ZL, Han FF, Peng XH, et al. YES-associated protein 1 promotes adenocarcinoma growth and metastasis through activation of the receptor tyrosine kinase Axl. Int J Immunopathol Pharmacol. 2012;25(4): 989-1001.

20. Zhou Z, Zhu JS, Xu ZP, Zhang Q. Lentiviral vector-mediated siRNA knockdown of the YAP gene inhibits growth and induces apoptosis in the SGC7901 gastric cancer cell line. Mol Med Rep. 2011;4(6):1075-1082.

21. Yu W, Qiao Y, Tang X, et al. Tumor suppressor long non-coding RNA, MT1DP is negatively regulated by YAP and Runx2 to inhibit FoxA1 in liver cancer cells. Cell Signal. 2014;26(12):2961-2968.
22. Gavine PR, Ren Y, Han L, et al. Volitinib, a potent and highly selective c-Met inhibitor, effectively blocks c-Met signaling and growth in c-MET amplified gastric cancer patient-derived tumor xenograft models. Mol Oncol. 2015;9(1):323-333.

23. Geng R, Li J. Apatinib for the treatment of gastric cancer. Expert Opin Pharmacother. 2015;16(1):117-122.

24. Sekiya T, Muthurajan UM, Luger K, Tulin AV, Zaret KS. Nucleosomebinding affinity as a primary determinant of the nuclear mobility of the pioneer transcription factor FoxA. Genes Dev. 2009;23(7):804-809.

25. Caravaca JM, Donahue G, Becker JS, He X, Vinson C, Zaret KS. Bookmarking by specific and nonspecific binding of FoxA1 pioneer factor to mitotic chromosomes. Genes Dev. 2013;27(3):251-260.

26. Imamura Y, Sakamoto S, Endo T, et al. FOXA1 promotes tumor progression in prostate cancer via the insulin-like growth factor binding protein 3 pathway. PLoS One. 2012;7(8):e42456.

27. Potter AS, Casa AJ, Lee AV. Forkhead box A1 (FOXA1) is a key mediator of insulin-like growth factor I (IGF-I) activity. J Cell Biochem. 2012;113(1):110-121.

28. Zhang Y, Tong T. FOXA1 antagonizes EZH2-mediated CDKN2A repression in carcinogenesis. Biochem Biophys Res Commun. 2014; 453(1):172-178.

29. Lim B, Park JL, Kim HJ, et al. Integrative genomics analysis reveals the multilevel dysregulation and oncogenic characteristics of TEAD4 in gastric cancer. Carcinogenesis. 2014;35(5):1020-1027.

30. Kang W, Tong JH, Chan AW, et al. Yes-associated protein 1 exhibits oncogenic property in gastric cancer and its nuclear accumulation associates with poor prognosis. Clin Cancer Res. 2011;17(8):2130-2139.

31. Hu X, Xin Y, Xiao Y, Zhao J. Overexpression of YAP1 is correlated with progression, metastasis and poor prognosis in patients with gastric carcinoma. Pathol Oncol Res. 2014;20(4):805-811.

32. Da CL, Xin Y, Zhao J, Luo XD. Significance and relationship between Yes-associated protein and survivin expression in gastric carcinoma and precancerous lesions. World J Gastroenterol. 2009;15(32): 4055-4061.

33. Jiao S, Wang $\mathrm{H}$, Shi Z, et al. A peptide mimicking VGLL4 function acts as a YAP antagonist therapy against gastric cancer. Cancer Cell. 2014;25(2): 166-180.
OncoTargets and Therapy

\section{Publish your work in this journal}

OncoTargets and Therapy is an international, peer-reviewed, open access journal focusing on the pathological basis of all cancers, potential targets for therapy and treatment protocols employed to improve the management of cancer patients. The journal also focuses on the impact of management programs and new therapeutic agents and protocols on

\section{Dovepress}

patient perspectives such as quality of life, adherence and satisfaction. The manuscript management system is completely online and includes a very quick and fair peer-review system, which is all easy to use. Visit http://www.dovepress.com/testimonials.php to read real quotes from published authors. 